\title{
Acute Kidney Injury in Transcatheter Aortic Valve Replacement
}

Sakina Abbas ${ }^{1}$, Ihtisham Qayum ${ }^{2}$, Rabiya Wahid ${ }^{3}$, FNU Salman ${ }^{4}$, Henna Khan ${ }^{5}$, Fatima Hassan ${ }^{6}$, Anum Babar $^{7}$, Arslan Inayat ${ }^{8}$

1. Medicine, Dow University of Health Sciences, Karachi, PAK 2. Internal Medicine, Khyber Teaching Hospital, Peshawar, PAK 3. Medicine, Liaquat University of Medical and Health Sciences, Hyderabad, PAK 4. Medicine, St. Vincent Medical Center, Toledo, USA 5. Internal Medicine, Khyber Girls Medical College, Peshawar, PAK 6. Medicine, Fatima Jinnah Medical University, Lahore, PAK 7. Medicine, Khyber Girls Medical College, Peshawar, PAK 8. Internal Medicine, University at Buffalo, Catholic Health System, Buffalo, USA

Corresponding author: Arslan Inayat, arslaninayat88@gmail.com

\section{Abstract \\ Introduction}

Transcatheter aortic valve replacement (TAVR) has been established as a standard of care for patients with severe aortic stenosis. We aim to study the predictors of acute kidney injury (AKI) after TAVR from a contemporary analysis using the National Inpatient Sample (NIS) database.

\section{Methods}

We performed a national analysis using the NIS database to evaluate predictors of acute kidney injury (AKI) after TAVR. Our study period was from 2015 to 2018, and we identified TAVR patients in all procedure fields. Patients aged less than 18 years were excluded from the study.

\section{Results}

We report data of 173,760 TAVR patients, of which 20,045 (11.5\%) had AKI and 153,715 (88.4\%) did not. There were three principal findings of our study. First, mortality was higher in patients with AKI compared to patients who did not have AKI ( $8 \%$ vs. $0.8 \%$; $p<0.01$ ). Second, patients with chronic kidney disease, weight loss, liver disease, congestive heart failure, cerebrovascular disease, chronic obstructive pulmonary disease, metastatic cancer, and peripheral vascular disease had higher adjusted odds of AKI. Third, length of stay and cost of stay were significantly higher in patients who had AKI during the index admission.

\section{Conclusion}

Patients with AKI had higher in-hospital mortality. We also report that at baseline, chronic kidney disease, weight loss, liver disease, congestive heart failure, cerebrovascular disease, chronic obstructive pulmonary disease, metastatic cancer, and peripheral vascular disease were important predictors of AKI in patients after TAVR. Length of stay and cost of stay were higher with AKI, which result in higher burden on the

Review began 05/08/2021 Review ended 05/15/2021 Published 05/21/2021

\section{() Copyright 2021}

Abbas et al. This is an open access article distributed under the terms of the Creative Commons Attribution License CC-BY 4.0., which permits unrestricted use, distribution, and reproduction in any medium, provided the original author and source are credited. health care system due to increased resource utilization.

Categories: Cardiac/Thoracic/Vascular Surgery, Cardiology

Keywords: tavr, tavi, aortic stenosis, transcatheter aortic valve replacement, transcatheter aortic valve implantation

\section{Introduction}

Transcatheter aortic valve replacement has revolutionized the treatment of patients with severe aortic stenosis who are considered high risk for surgical aortic valve replacement [1-4]. The PARTNER (Placement of AoRTic TraNscathetER Valves) trials have led the way for broadening the indication of TAVR to low-risk surgical patients [3]. TAVR has led to a constant improvement in clinical outcomes with aortic stenosis [5]. In patients with aortic valve disease, TAVR, in the last 15 years has progressed from a last resort procedure in patients who were at high perioperative risk for major mortality and morbidity from surgical valve replacement to a viable and alternate option to surgery [6]. Although TAVR has shown great results, acute kidney injury (AKI) remains one of the major complications and is associated with mortality, increased adverse events, and resource use $[7,8]$.

Rapid loss of kidney function occurring within hours or days and resulting in impaired electrolyte hemostasis, dysregulation of volume, and accumulation of waste product is defined as AKI [9]. AKI is a frequent complication seen in TAVR that remains associated with a dismal prognosis and was identified as one of the most common complications in the landmark PARTNER trials [2,3,10-12]. According to one estimate, AKI is seen in up to 30\% of patients undergoing TAVR [13]. We know from prior literature that AKI in surgical aortic valve replacement was associated with up to a four-fold higher risk of mortality. During the 
procedure, there are multiple predisposing factors such as contrast use and hypotension episodes that predispose to acute renal injury [14]. As TAVR is mostly performed in elderly and frail patients, CKD has a wide prevalence and further predisposes patients to acute-on-chronic kidney injury [12,14-18].

Previous studies have looked at AKI in TAVR; however, studies have remained limited to case series and retrospective studies with a small sample size. Predictors of AKI after TAVR from a large sample size of patients and real-world experience of TAVR remain scarce. Hence, the aim of this study was to identify important predictors of AKI, mortality rate, and resource utilization after TAVR from a U.S. national database.

\section{Materials And Methods}

We used the U.S. National Inpatient Sample (NIS) database to identify cases of TAVR performed from 2015 to 2018. The NIS data are available to the public and anonymized; hence, Institutional Review Board approval was not necessary for this study.

To perform a national analysis, we used the International Classification of Diseases (ICD)- 9 and ICD-10 codes $(3505,3506$, and O2RF3) to identify hospitalizations for TAVR. We queried all diagnosis fields to select TAVR patients. Similarly, ICD-9 and ICD-10 diagnosis codes were used to define and identify all baseline comorbidities. We excluded patients who were less than 18 years of age.

We used weighted data based on discharge weights provided by the NIS. For categorical variables, we used the chi-square test. For continuous variables, testing of non-normality was used. Since continuous variables are not normally distributed, Mann-Whitney U test was used. We also developed a binary logistic model using entry method including demographic factors such as age, sex, race, median income, hospital location, baseline co-morbidities, obesity, weight loss, metastatic cancer, lymphoma, solid organ tumor, alcohol use, coagulopathy, hypothyroidism, chronic obstructive pulmonary disease (COPD), cerebrovascular disease (CVA), congestive heart failure (CHF), coronary artery disease (CAD), diabetes mellitus, hypertension, liver disease, chronic kidney disease (CKD), and peripheral vascular disease (PVD). In compliance with the Healthcare Cost and Utilization Project guidelines, we did not report observations with less than 11 cases. Comparisons were two-sided, and $\mathrm{p}<0.05$ was considered statistically significant. All analyses were performed utilizing SPSS Version 27 (IBM Corp., Armonk, NY) and R Version 3.5 (R Foundation for Statistical Computing, Vienna, Austria).

\section{Results}

A total of 173,760 weighted hospitalizations for TAVR were included in the analysis. Of the patient undergoing the procedure, 20,045 (11.5\%) had AKI and 153,715 (88.4\%) did not. The detailed baseline characteristics are summarized in Table1. At baseline, patients with CKD (OR: 3.52; 95\% CI: 3.40-3.64), weight loss (OR: 3.01; 95\% CI: 2.82-3.20), liver disease (OR: 2.29; 95\% CI: 2.13-2.46), CHF (OR: 2.01; 95\% CI: 1.92-2.10), CVA (OR: 1.30; 95\% CI: 1.24-1.36), COPD (OR: 1.21; 95\% CI: 1.17-1.25), metastatic cancer (OR: 1.16; 95\% Cl: 0.95-1.41), and PVD (OR: 1.15; 95\% CI: 1.11-1.19) had higher adjusted odds of AKI (Table1, Figure 1). Mortality rate was higher with AKI ( $8 \%$ vs $0.8 \%$; $<0.01)$. Patients who had AKI had a higher median cost of stay (US $\$ 63,110$ vs. US $\$ 44,853 ; \mathrm{p}<0.01$ ) and length of stay ( 9 vs. 2 days; $\mathrm{p}<0.01$ ) (Table 2). 


\section{Cureus}

\begin{tabular}{|c|c|c|c|}
\hline Variable & $\begin{array}{l}\text { No AKI } \\
(153,715)\end{array}$ & With AKI $(20,045)$ & No AKI vs. AKI (multivariate analysis), OR (95\% Cl) \\
\hline Age, median (IQR) & $82(75-86)$ & $82(74-87)$ & $1.00(0.97-1.04)$ (reference age $<75$ years) \\
\hline Male gender & $81590(53.1 \%)$ & 11585 (57.8\%) & 1.12 (1.08-1.16) (reference to female) \\
\hline Caucasian & $128800(87.4 \%)$ & 15875 (83.4\%) & Reference \\
\hline African Americans & $6060(4.1 \%)$ & $1005(5.3 \%)$ & $0.74(0.67-0.81)$ \\
\hline Hispanics & $6855(4.6 \%)$ & $1285(6.7 \%)$ & $0.71(0.63-0.80)$ \\
\hline Chronic kidney disease & $33645(21.9 \%)$ & $10575(52.8 \%)$ & $3.52(3.40-3.64)$ \\
\hline Weight loss & $3870(2.5 \%)$ & $1865(9.3 \%)$ & $3.01(2.82-3.20)$ \\
\hline Liver disease & $4060(2.6 \%)$ & $1405(7.0 \%)$ & $2.29(2.13-2.46)$ \\
\hline Congestive heart failure & $110385(71.8 \%)$ & 17205 (85.8\%) & $2.01(1.92-2.10)$ \\
\hline Coagulopathy & $17270(11.2 \%)$ & $4315(21.5 \%)$ & $1.82(1.75-1.90)$ \\
\hline Cerebrovascular disease & $17320(11.3 \%)$ & $2930(14.6 \%)$ & $1.30(1.24-1.36)$ \\
\hline Solid organ tumor & $3655(2.4 \%)$ & $605(3.0 \%)$ & $1.23(1.11-1.36)$ \\
\hline Chronic obstructive pulmonary disease & $46145(30.0 \%)$ & 7095 (35.4\%) & $1.21(1.17-1.25)$ \\
\hline Metastatic cancer & $995(0.6 \%)$ & $150(0.7 \%)$ & $1.16(0.95-1.41)$ \\
\hline Peripheral vascular disease & $33015(21.5 \%)$ & $5130(25.6 \%)$ & $1.15(1.11-1.19)$ \\
\hline Obesity & $25870(16.8 \%)$ & $3335(16.6 \%)$ & $1.01(0.97-1.05)$ \\
\hline Lymphoma & $1060(0.7 \%)$ & $125(0.6 \%)$ & $0.99(0.81-1.20)$ \\
\hline Alcohol use & $240(0.2 \%)$ & $45(0.2 \%)$ & $0.98(0.70-1.39)$ \\
\hline Hypothyroidism & $31350(20.4 \%)$ & $4050(20.2 \%)$ & $0.98(0.94-1.02)$ \\
\hline Coronary artery disease & $106475(69.3 \%)$ & 13950 (69.6\%) & $0.92(0.89-1.00)$ \\
\hline Hypertension & $136605(88.9 \%)$ & $18015(89.9 \%)$ & $0.90(0.85-1.0)$ \\
\hline Diabetes mellitus & 25140 (16.4\%) & 1990 (9.9\%) & $0.76(0.72-0.80)$ \\
\hline \multicolumn{4}{|l|}{ Income } \\
\hline $0-25$ th percentile & $32110(21.2 \%)$ & $4485(22.7 \%)$ & Reference \\
\hline 25th-50th percentile & $38530(25.4 \%)$ & $5210(26.4 \%)$ & $1.08(1.03-1.13)$ \\
\hline 50th-75th percentile & $40805(26.9 \%)$ & $4955(25.1 \%)$ & $1.05(1.00-1.10)$ \\
\hline 75th-100th percentile & 39990 (26.4\%) & $5075(25.7 \%)$ & $0.93(0.89-0.97)$ \\
\hline Urban & $1380(0.9 \%)$ & 110 (0.5\%) & Reference \\
\hline Urban non-teaching & $14550(9.5 \%)$ & $1625(8.1 \%)$ & $0.56(0.46-0.69)$ \\
\hline Urban teaching & $137785(89.6 \%)$ & $18310(91.3 \%)$ & $0.85(0.80-0.9$ \\
\hline
\end{tabular}

TABLE 1: Baseline characteristics and predictors of AKI in patients after TAVR

AKI, acute kidney injury; IQR, interquartile range; TAVR, transcatheter aortic valve replacement 


\section{Cureus}

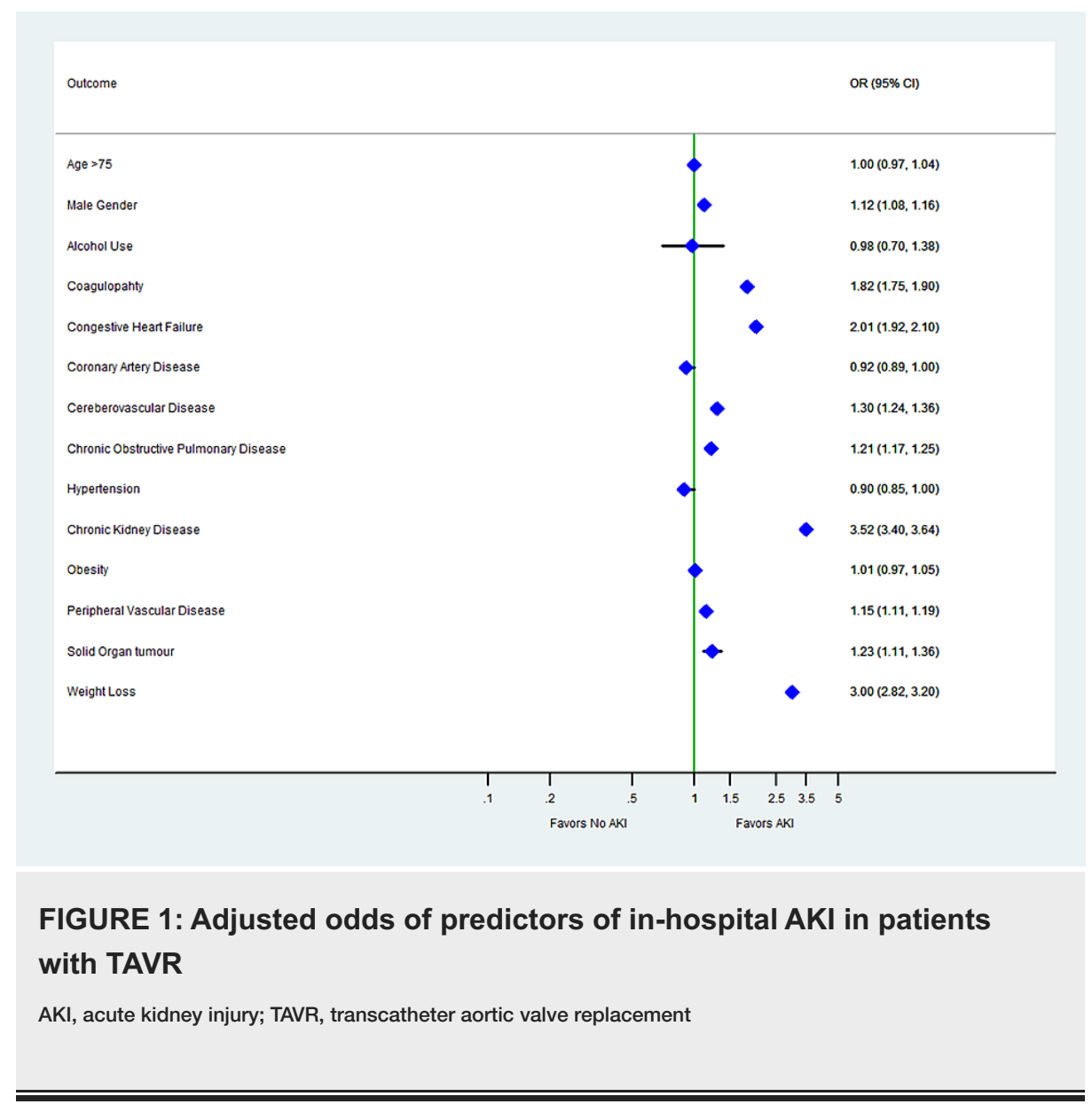

\begin{tabular}{|c|c|c|c|}
\hline Outcome & No AKI & AKI & p-Value \\
\hline Died during hospitalization, n (\%) & $1285(0.8 \%)$ & $1605(8 \%)$ & $<0.01$ \\
\hline Length of stay, median (IQR) & $2(2-4)$ & $9(5-15)$ & $<0.01$ \\
\hline Cost of stay (in US dollars), median (IQR) & $44853(35218-56473)$ & $63110(47235-85623)$ & $<0.01$ \\
\hline
\end{tabular}

\section{TABLE 2: In-hospital outcomes of patients with AKI in TAVR}

AKI, acute kidney injury; IQR, interquartile range; TAVR, transcatheter aortic valve replacement

\section{Discussion}

The principal findings of our study are summarized as follows: (1) AKI occurs in about $11.5 \%$ of patients undergoing TAVR, (2) mortality is higher in patients with AKI compared to patients who do not have AKI (8\% vs. $0.8 \%$; $\mathrm{p}<0.01$ ), (3) factors at baseline such as male sex, CVA, CHF, COPD, liver disease, CKD, metastatic cancer, weight loss, and PVD are important predictors of AKI in patients undergoing TAVR, and (4) patients who developed AKI after TAVR have a higher length of stay and cost of stay.

The reported incidence of post-TAVR AKI is $22.1 \% \pm 11.2 \%$ based on the current Valve Academic Research Consortium (VARC)-2. Both persistent and transient AKI have been independently associated with higher mortality rates. Hence, our study aims to identify baseline characteristics that predispose a patient to AKI so that we can institute an approach of pre-TAVR intravenous fluid hydration and try to minimize contrast exposure in these patients [9]. In contrast to the previous literature, in our national analysis, we have reported an AKI rate of $13 \%$. However, it is important to note that we used ICD codes to identify AKI, whereas prior literature has reported AKI in terms of VARC-2 criteria [12]. However, our study results were congruent with the findings of Bagur et al., who reported an AKI rate of $11.7 \%$, which is very close to our estimate of $13 \%$. In prior literature, mortality rate in TAVR was approximated to be $28 \%$, which was significantly higher compared to our study findings (8\%) [14]. One of the possible explanations is that our study was more recent and has looked at TAVR experience in recent times, which have undergone significant 
technological advancements and increased operator experience. Similarly, Aregger et al. reported data on a series of 54 patients and reported an AKI frequency of 28\% [19]. In contrast, our study reported more new findings from a large sample size of 173,760 patients, which would be a better indicator of AKI complication rates in recent times.

According to one estimate, patients who developed AKI had a four-fold increase in mortality. Kidney disease is a well-known predictor of worse outcomes in patients undergoing TAVR [12,20,21]. Our study findings are consistent with prior literature, and we report that patients with CKD have a 3.5 times higher risk of developing AKI. Similarly, co-morbidities such as COPD have been reported in prior literature to cause AKI [22]. Our study reinforces these findings and reports a 1.12-fold higher risk of AKI with COPD. Similarly, our national analysis identifies CVA, liver disease, and PVD as significant predictors of this adverse event. Previous studies have reported hypertension to be an important predictor of AKI due to loss of kidney autoregulation. However, in our analysis, hypertension was not found to be significantly associated with an increased incidence of AKI [23].

Our study findings must be interpreted in light of the following limitations. This was a retrospective study hence association should not be misinterpreted as causation. Important data including medication use and laboratory data were not available in the NIS. Surgical risk scores such as EuroSCORE (European System for Cardiac Operative Risk Evaluation) and STS (Society of Thoracic Surgeons) score cannot be calculated using this database. We were only able to look at in-hospital data, and longer follow-up data were not available. NIS is a billing dataset that relies on ICD codes; hence, coding errors are a possibility. CKD in our study was defined as CKD-1 to CKD-IV based on ICD-9 and ICD-10 diagnosis codes. We used ICD codes whereas nondatabase studies used standardized definitions. However, we used well-validated ICD codes that reduce the chance of coding errors. We are not able to quantify the degree of AKI from the NIS database given its inherent limitations; in contrast, previous studies used VARC-2 criteria for AKI definition and were able to quantify AKI.

\section{Conclusions}

In conclusion, our study reported a contemporary analysis of 173,760 TAVR patients. The rate of AKI postTAVR was $11.5 \%$. We further reported that at baseline CKD, weight loss, liver disease, CHF, CVA, COPD, metastatic cancer, and PVD were important predictors of AKI in patients undergoing TAVR. AKI in TAVR is associated with increased burden on the healthcare system in the form of increased length of hospital stay and cost of hospitalization. It is of utmost importance to identify patients who are at high risk of developing complications as TAVR indication expands to a larger population. These data can also help clinicians in decision-making to optimize patients prior to the procedure and decrease the risk of AKI post-TAVR.

\section{Additional Information \\ Disclosures}

Human subjects: Consent was obtained or waived by all participants in this study. N/A issued approval N/A. Given the deidentified nature of the NIS database, IRB approval is not required. Animal subjects: All authors have confirmed that this study did not involve animal subjects or tissue. Conflicts of interest: In compliance with the ICMJE uniform disclosure form, all authors declare the following: Payment/services info: All authors have declared that no financial support was received from any organization for the submitted work. Financial relationships: All authors have declared that they have no financial relationships at present or within the previous three years with any organizations that might have an interest in the submitted work. Other relationships: All authors have declared that there are no other relationships or activities that could appear to have influenced the submitted work.

\section{References}

1. Rotman OM, Bianchi M, Ghosh RP, Kovarovic B, Bluestein D: Principles of TAVR valve design, modelling, and testing. Expert Rev Med Devices. 2018, 15:771-9. 10.1080/17434440.2018.1536427

2. Smith CR, Leon MB, Mack MJ, et al.: Transcatheter versus surgical aortic-valve replacement in high-risk patients. N Engl J Med. 2011, 364:2187-98. 10.1056/NEJMoa1103510

3. Mack MJ, Leon MB, Thourani VH, et al.: Transcatheter aortic-valve replacement with a balloon-expandable valve in low-risk patients. N Engl J Med. 2019, 380:1695-70. 10.1056/NEJMoa1814052

4. Ullah W, Sattar Y, Al-Khadra Y, et al.: Clinical outcomes of renal and liver transplant patients undergoing transcatheter aortic valve replacement: analysis of national inpatient sample database. Expert Rev Cardiovasc Ther. 2021, 19:363-8. 10.1080/14779072.2021.1892489

5. Tchetche D, de Biase C, Brochado B, Mastrokostopoulos A: How to make the TAVI pathway more efficient. Interv Cardiol. 2019, 14:31-3. 10.15420/icr.2018.28.2

6. Cormican D, Jayaraman A, Villablanca P, Ramakrishna H: TAVR procedural volumes and patient outcomes: analysis of recent data. J Cardiothorac Vasc Anesth. 2020, 34:545-50. 10.1053/i.jvca.2019.04.016

7. Zaleska-Kociecka M, Dabrowski M, Stepinska J: Acute kidney injury after transcatheter aortic valve replacement in the elderly: outcomes and risk management. Clin Interv Aging. 2019, 14:195-201. 10.2147/CIA.S149916

8. Grube E, Sinning JM: The "big five" complications after transcatheter aortic valve replacement: do we still have to be afraid of them?. JACC Cardiovasc Interv. 2019, 12:370-2. 10.1016/j.jcin.2018.12.019 
9. Scherner M, Wahlers T: Acute kidney injury after transcatheter aortic valve implantation . J Thorac Dis. 2015, 7:1527-35. 10.3978/j.issn.2072-1439.2015.06.14

10. Arora S, Misenheimer JA, Ramaraj R: Transcatheter aortic valve replacement: comprehensive review and present status. Tex Heart Inst J. 2017, 44:29-38. 10.14503/THIJ-16-5852

11. Peillex M, Marchandot B, Bayer S, et al.: Bedside renal Doppler ultrasonography and acute kidney injury after TAVR. J Clin Med. 2020, 9:905. 10.3390/jcm9040905

12. Alassar A, Roy D, Abdulkareem N, Valencia O, Brecker S, Jahangiri M: Acute kidney injury after transcatheter aortic valve implantation: incidence, risk factors, and prognostic effects. Innovations (Phila). 2012, 7:389-93. 10.1097/IMI.0b013e3182814e43

13. Rosner MH, Okusa MD: Acute kidney injury associated with cardiac surgery . Clin J Am Soc Nephrol. 2006, 1:19-32. 10.2215/CJN.00240605

14. Bagur R, Webb JG, Nietlispach F, et al.: Acute kidney injury following transcatheter aortic valve implantation: predictive factors, prognostic value, and comparison with surgical aortic valve replacement. Eur Heart J. 2010, 31:865-74. 10.1093/eurheartj/ehp552

15. Sardar MR, Goldsweig AM, Abbott JD, Sharaf BL, Gordon PC, Ehsan A, Aronow HD: Vascular complications associated with transcatheter aortic valve replacement. Vasc Med. 2017, 22:234-4. 10.1177/1358863X17697832

16. Patel KV, Omar W, Gonzalez PE, Jessen ME, Huffman L, Kumbhani DJ, Bavry AA: Expansion of TAVR into low-risk patients and who to consider for SAVR. Cardiol Ther. 2020, 9:377-94. 10.1007/s40119-020-00198-Z

17. Ullah W, Zahid S, Hamzeh I, Birnbaum Y, Virani SS, Alam M: Trends and predictors of transcatheter aortic valve implantation related in-hospital mortality (from the National Inpatient Sample Database). Am J Cardiol. 2021, 143:97-103. 10.1016/j.amjcard.2020.12.031

18. Ullah W, Jafar M, Zahid S, et al.: Predictors of in-hospital mortality in patients with end-stage renal disease undergoing transcatheter aortic valve replacement: a nationwide inpatient sample database analysis [Online ahead of print]. Cardiovasc Revasc Med. 2021, 10.1016/j.carrev.2021.02.002

19. Aregger F, Wenaweser P, Hellige GJ, Kadner A, Carrel T, Windecker S, Frey FJ: Risk of acute kidney injury in patients with severe aortic valve stenosis undergoing transcatheter valve replacement. Nephrol Dial Transplant. 2009, 24:2175-9. 10.1093/ndt/gfp036

20. Anand IS, Chandrashekhar Y, Ferrari R, et al.: Pathogenesis of congestive state in chronic obstructive pulmonary disease. Studies of body water and sodium, renal function, hemodynamics, and plasma hormones during edema and after recovery. Circulation. 1992, 86:12-21. 10.1161/01.cir.86.1.12

21. Zahid S, Ullah W, Khan MU, et al.: Trends, predictors, and outcomes of major bleeding after transcatheter aortic valve implantation, from National Inpatient Sample (2011-2018) [Online ahead of print]. Expert Rev Cardiovasc Ther. 2021, 10.1080/14779072.2021.1924678

22. Ando T, Adegbala O, Akintoye E, et al.: Is transcatheter aortic valve replacement better than surgical aortic valve replacement in patients with chronic obstructive pulmonary disease? A Nationwide Inpatient Sample analysis. J Am Heart Assoc. 2018, 7:e008408. 10.1161/JAHA.117.008408

23. Hsu CY, Ordoñez JD, Chertow GM, Fan D, McCulloch CE, Go AS: The risk of acute renal failure in patients with chronic kidney disease. Kidney Int. 2008, 74:101-7. 10.1038/ki.2008.107 